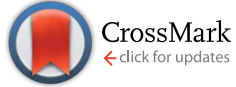

Cite this: RSC Adv., 2015, 5, 27874

Received 14th January 2015

Accepted 2nd March 2015

DOI: 10.1039/c5ra00819k

www.rsc.org/advances

\section{Graphene-based structural adhesive to enhance adhesion performance}

\author{
Liberata Guadagno, ${ }^{\star a b}$ Maria Sarno, ${ }^{\text {ab }}$ Umberto Vietri, ${ }^{a}$ Marialuigia Raimondo, ${ }^{a}$ \\ Claudia Cirillo ${ }^{\mathrm{ab}}$ and Paolo Ciambellia
}

\begin{abstract}
This paper proposes the design of a new graphene nano-modified formulation to enhance the mechanical performance of structural adhesives. Well-characterized graphene platelets, produced through an effective approach for bulk production and morphology control, were embedded at different contents inside an epoxy adhesive based on tetraglycidylmethylene dianiline (TGMDA). The adhesive formulations were used to manufacture bonded joints, according to ASTM 2095, to analyze the effect of graphene platelets on the tensile strength of the joints. The effect of incorporating graphene in the adherents was also considered. Epoxy adhesives filled with graphene at a concentration of 1 wt $\%$ significantly enhanced the mechanical behavior of the bonded joints. Only in the case of unfilled adherents, the inclusion of $4 \mathrm{wt} \%$ graphene did not have a significant effect on the mechanical performance. This is likely due to the agglomeration of nanofillers causing heterogeneity in large domains at the interface between adherents and adhesives. The effect of graphene incorporation in the adherents, acting on the chemical compatibility between adhesives and adherent surfaces, led to a considerable increase in tensile strength in comparison with the corresponding joints with unfilled adherents. This beneficial effect is most probably due to the cumulative effects of intermolecular interactions between the graphene platelets and resin networks.
\end{abstract}

\section{Introduction}

Adhesively bonded joints are attracting increasing interests to be alternatives to mechanical joints in engineering applications, and they provide many advantages over conventional mechanical seals. Adhesive bonding provides structural design possibilities, which cannot be obtained by any other technique. Furthermore, in most of the load cases (namely, tension, compression, and shear), the adhesively bonded structures are characterized by lower weights. Bonded joints are gas and moisture tight (unlike riveted or bolted joints) with good durability, excellent resistance against corrosion and good fatigue properties. Currently, structural bonding is still not a widely accepted alternative to riveting due to doubts about their reliability in terms of mechanical performance and long-term bonding capability. This has led, in many structural applications, to the introduction of fail-secure devices that in many cases are very disadvantageous in terms of weight, application and cost. Therefore, the current challenge is to overcome drawbacks such as the poor mechanical performance, the electrical insulating properties, water sorption and debonding,

${ }^{a}$ Department of Industrial Engineering, DIIn, University of Salerno, via Giovanni Paolo II,132,84084 Fisciano, SA, Italy.E-mail: lguadagno@unisa.it

${ }^{b}$ Centre NANO_MATES, University of Salerno, via Giovanni Paolo II,132, 84084 Fisciano, SA, Italy flammability and critical points related to the processing temperature. In this paper, a formulation designed for a structural adhesive to enhance the mechanical performance of epoxy adhesives nano-modified by graphene is presented. Graphene platelets were embedded inside an epoxy adhesive based on a mixture of tetraglycidylmethylene dianiline (TGMDA) and 1,4-butanedioldiglycidylether (BDE). This particular epoxy formulation has proven to be very effective for improving nanofiller dispersions due to a decrease in viscosity; ${ }^{\mathbf{1}, 2}$ in addition, it was determined that it allows to reduce the moisture content, which is a very critical point for adhesive bonding. The chemical composition of this epoxy formulation reduces the equilibrium sorption of liquid water $\left(C_{\text {eq }}\right)$ by about $35 \% .^{3,4}$ This is a very large value in the application of epoxy mixtures as structural adhesive materials; for example, in aeronautics, the absorbed moisture reduces the matrix-dominated mechanical properties. The absorbed moisture also causes the adhesive to swell, and during freeze-thaw cycles, it expands during freezing and can crack the material. The particular epoxy matrix also proved to be beneficial for improving the cure degree. It increases the mobility of the chains containing the reactive groups, resulting in a higher cure degree than the epoxy precursor alone. ${ }^{4}$

Several papers have been published on the inclusion of nano-structured carbon forms, including graphene, inside epoxy adhesives to enhance the mechanical strength and 
toughness of the bonded joints. ${ }^{5-9}$ In addition, it is well known that one of the main predicted advantages related to the inclusion of conductive nanoparticles into an epoxy resin is the improvement of its electrical behavior. ${ }^{\mathbf{1 0 - 1 6}}$ Different types of carbon nanofillers are electrical conductor materials, which if well dispersed in the matrix, can drastically increase the electrical properties of epoxy based adhesives even at a very low percentage of nanofillers, moreover graphene in particular exhibits fascinating physical properties such as thermodynamic stability, extremely high charge carrier mobility and mechanical stiffness, which are critical requisites for several applications. ${ }^{17-23}$ Recently, Bunch et al. highlighted another relevant feature of graphene sheets; atomic membranes from graphene sheets are impermeable to standard gases, including helium, and they can withstand pressure differences larger than one atmosphere. ${ }^{24}$ We would expect a similar performance for a bulk polymer containing homogenously dispersed graphene sheets. This outstanding feature is of potential interest in the field of structural adhesives. Moreover, graphene based materials can activate self-assembling mechanisms that are able to improve the gas barrier and mechanical properties of nanocomposites..$^{25,26}$

The enhancement of the different properties of epoxy-based materials and/or adhesives depends on numerous parameters, such as the chemical nature of nanofiller, adhesive and adherents, the applied surface treatment and the tested properties. ${ }^{16}$ Concerning the chemical nature of the nanofiller, the structure and different morphological parameters, which are strongly dependent on the preparation procedure, may vary in a wide range of values; thus, different types of effects on the adhesive performance can be determined. For this reason, graphene, which was well characterized and prepared using an approach for bulk production and morphology control, has been used in this work.

Since the first successful fabrication of graphene by cleavage, three major methods for the fabrication of single- and multilayer graphene have been investigated. These methods include micromechanical cleavage, ${ }^{17}$ exfoliation of graphite ${ }^{27,28}$ and chemical vapor deposition (CVD) of hydrocarbons on a substrate surface. ${ }^{20,29-33}$ Among these, the exfoliation of graphite is observed to be a considerably more efficient approach for the bulk production of graphene platelets.

Moreover, several papers involve the exfoliation of graphene oxide (GO). ${ }^{19,34,35}$ This material consists of graphene-like platelets, as well as chemically functionalized with compounds such as hydroxyls and epoxides, which stabilize the platelets in water. ${ }^{27}$ However, GO has poor quality and the required reduction often results in the generation of a significant number of defects. ${ }^{27,34}$ However, defect-free, unoxidized graphene can be obtained by a non-covalent, solution-phase method. ${ }^{28}$ Hernandez et al. obtained a high-quality monolayer graphene with significant yields (1 wt\%) via a non-chemical, solution-phase exfoliation by sonicating graphite in an organic solvent. Graphene used for this work was prepared by a liquid phase exfoliation in $N$-methylpyrrolidone (NMP). The sonication parameters have been modulated to treat an elevated graphite concentration solution to obtain a high graphene yield with a one-step massive production of thin platelets.

The incorporation of graphene platelets, prepared using the abovementioned procedure, on the adhesive performance was studied. A suitable fraction of graphene platelets inside an epoxy paste leads to an enhancement in joint strength. An epoxy matrix nanofilled up to $1 \mathrm{wt} \%$ significantly enhanced the mechanical behavior of the bonded joints, whereas the addition of $4 \mathrm{wt} \%$ graphene exhibited a non-beneficial effect on the adhesive performance probably due to the agglomeration of nanofillers, which is responsible for the destruction of the continuity of the morphological features of the adhesive. The incorporation of graphene platelets in the adherents led to a considerable increment in tensile strength in comparison with the corresponding joints with unfilled adherents.

\section{Experimental section}

\subsection{Materials}

Epoxy matrix. An epoxy matrix was prepared by mixing an epoxy precursor, namely, TGMDA (epoxy equivalent weight 117$133 \mathrm{~g} \mathrm{eq}^{-1}$ ), with an epoxy reactive monomer 1,4-butanedioldiglycidylether (BDE), which acts as a reactive diluent. These resins, both containing an epoxy group, were obtained from Sigma-Aldrich. The curing agent investigated for this study is $4,4^{\prime}$-diaminodiphenyl sulfone (DDS). A hardener was used at a stoichiometric concentration with respect to oxirane rings. In this paper, the epoxy mixture is labeled as T20BD.

Nanofiller. Graphite powder was purchased from Alfa Aesar (microcrystalline, -300 mesh). $N$-Methylpyrrolidone was purchased from Sigma-Aldrich (spectrophotometric grade $>99.0 \%)$.

\subsection{Sample preparation}

Graphene. Graphene platelets were prepared by the liquid phase exfoliation of graphite. Graphite was dispersed in $N$ methylpyrrolidone (NMP) at a concentration of $10 \mathrm{mg} \mathrm{ml}^{-1}$ and sonicated for $1 \mathrm{~h}$. This method facilitates the production of high-quality, unoxidized graphite and graphene platelets from powdered graphite. In this manner, colloidal dispersions of graphene platelets are obtained through the exfoliation of graphite in the liquid phase in suitable organic solvents. This is possible because the energy required to exfoliate graphene is balanced by the solvent-graphene interaction, particularly for solvents whose surface energies match that of graphene. Different techniques were used to demonstrate the presence of an individual graphene and the distribution of the thickness of the platelets (such as transmission electron microscopy, electron diffraction study, Raman spectroscopy, and thermogravimetric analysis).

The best result, which was obtained by dispersing graphite in $N$-methylpyrrolidone (NMP, spectrophotometric grade $>99.0 \%$ ) (cylindrical vial, 10-25 ml solvent) at a concentration of $10 \mathrm{mg} \mathrm{ml}^{-1}$ for $1 \mathrm{~h}$ at the maximum power for ultrasonication (Hielscher UP 400S), is reported in the following sections. The product obtained by sonication was a liquid consisting of a 
greyish homogeneous phase, containing a number of macroscopic aggregates that can be removed by centrifugation for 90 minutes at $500 \mathrm{rpm}$.

Graphene and multilayer graphene platelets (G + MLG) were recovered by vacuum filtration of the supernatant solution, obtained by centrifugation, onto a $20 \mathrm{~nm}$ pore size alumina membrane. The supernatant contained about $30 \mathrm{wt} \%$ of the original graphite (see TG evaluation under Results and discussion section).

Adherents and adhesive. In this study, 0.5\%, 1\% and 4\% wt/ wt of graphene was dispersed in an epoxy resin to obtain an adhesive formulation to bond unfilled and filled epoxy adherents. Epoxy blend and DDS were mixed at $120{ }^{\circ} \mathrm{C}$ and the graphene platelets were added and incorporated into the matrix by ultrasonication for $20 \mathrm{~min}$. An ultrasonic device, Hielschermodel UP200S (200 W, $24 \mathrm{kHz})$, was used. The combinations are shown in Table 1.

Adhesive formulations were cured by a two-stage curing cycle: an initial step at a moderate temperature $\left(125^{\circ} \mathrm{C}\right.$ for $\left.1 \mathrm{~h}\right)$ and the second one at a higher temperature $\left(180{ }^{\circ} \mathrm{C}\right.$ for $\left.3 \mathrm{~h}\right)$.

\subsection{Characterization techniques}

Field emission scanning electron microscopy (FESEM) images were captured with a LEO 1525 microscope. Transmission electron microscopy (TEM) images were acquired with an FEI Tecnai electron microscope operating at $200 \mathrm{kV}$ with a $\mathrm{LaB}_{6}$ filament as the source of electrons. The samples for the TEM observation were casted from an NMP solution on the TEM grid.

The Raman spectra of both the supernatant and precipitate from centrifugation, casting on a glass slip and after NMP drying, were obtained at room temperature with a microRaman spectrometer (Renishaw inVia; $514 \mathrm{~nm}$ excitation wavelength). For all the samples, about 100 measurements have been carried out. The laser spot diameter was $10 \mu \mathrm{m}$, which is a value higher than the size of the platelets obtained.

X-ray diffraction (XRD) measurements were performed with a Bruker D8 X-ray diffractometer using $\mathrm{CuK} \alpha$ radiation. The measurements were performed on a thin carbon film obtained after filtration.

Thermal decomposition behaviour was investigated with a thermo-analyzer (Q600, TA Instruments), which was connected online to a quadrupole mass detector (Quadstar 422, Pfeiffer Vacuum). The measurement was carried out in air.
The adhesive performance of the nanofilled epoxy-based materials was investigated considering bonded joint configurations, which are based on the ASTM D 2095 standard.

Materials (unfilled and filled epoxy mixture) were cured in an appropriate mold geometry configuration made of Teflon (PTFE). The mold was designed following the existing international standard practice for the design of the specimens; in particular, ASTM D 2094 and ASTM D 1002 were considered. In this manner, a suitable configuration of specimens for tensile butt joint (referred to ASTM D 2095) was obtained to measure the mechanical strength, and thus the adhesion properties of the bonded joint (see Scheme 1).

FESEM investigation was carried out using an FESEM instrument (FE-SEM, mod. LEO 1525, Carl Zeiss SMT AG, Oberkochen, Germany) with the aim of studying the morphology of the detached zones. The fractured surfaces of the bonding areas were preliminary coated with a thin gold layer of thickness $250 \AA$.

Mechanical testing. Adhesive tests were carried out using an electro-hydraulic servo-controlled testing machine (Instron mod. 4301). The tensile strength of the nano-reinforced interface was measured. In tensile butt joint tests, the specimens were placed in the testing machine and the loading was applied at a rate of $20 \mathrm{MPa} \mathrm{cm}{ }^{-2}$ of bond area per min $\left(3.1 \mathrm{kN} \mathrm{min}^{-1}\right)$ according to the ASTM D 2095 standard requirement; a load cell of $5 \mathrm{kN}$ was required for this purpose. This arrangement also ensures that no slip would take place during the test. Five samples were prepared for each set, as required by ASTM D 2095 , and average values of the mechanical strength of the joints were measured. For this purpose, the maximum tensile load measured during the tests was divided by the transversal bonded area. The elastic modulus of the adhesive paste was determined by the tensile test on bulk specimens (ASTM D 638 standard requirements). Several samples were tested for each set in the tensile mode by applying a loading rate of $1 \mathrm{~mm} \mathrm{~min}^{-1}$. In any case, Young's modulus was calculated as the slope of the stress-strain curve in the linear region.

\section{Results and discussion}

\subsection{Graphene characterization}

FESEM images of the obtained platelets with increase in magnification are shown in Fig. 1; the powder consists of nanometer-sized platelets.

Table 1 Summary of the prepared samples

\begin{tabular}{|c|c|c|c|}
\hline Sample label & Adherent & Adhesive & $\begin{array}{l}\text { Adhesive thickness } \\
{[\mathrm{mm}] \text { tensile butt joint }}\end{array}$ \\
\hline A & T20BD & $\mathrm{T} 20 \mathrm{BD}$ & $0.19 \pm 0.15$ \\
\hline B & T20BD & T20BD/0.5\% wt/wt graphene & $0.2 \pm 0.05$ \\
\hline $\mathrm{C}$ & T20BD & T20BD/1\% wt/wt graphene & $0.15 \pm 0.1$ \\
\hline $\mathrm{D}$ & T20BD & T20BD/4\% wt/wt graphene & $0.18 \pm 0.05$ \\
\hline $\mathrm{E}$ & T20BD/0.5\% wt/wt graphene & T20BD/0.5\% wt/wt graphene & $0.15 \pm 0.05$ \\
\hline $\mathrm{F}$ & T20BD/1\% wt/wt graphene & T20BD/1\% wt/wt graphene & $0.18 \pm 0.05$ \\
\hline G & T20BD/4\% wt/wt graphene & T20BD/4\% wt/wt graphene & $0.15 \pm 0.15$ \\
\hline
\end{tabular}



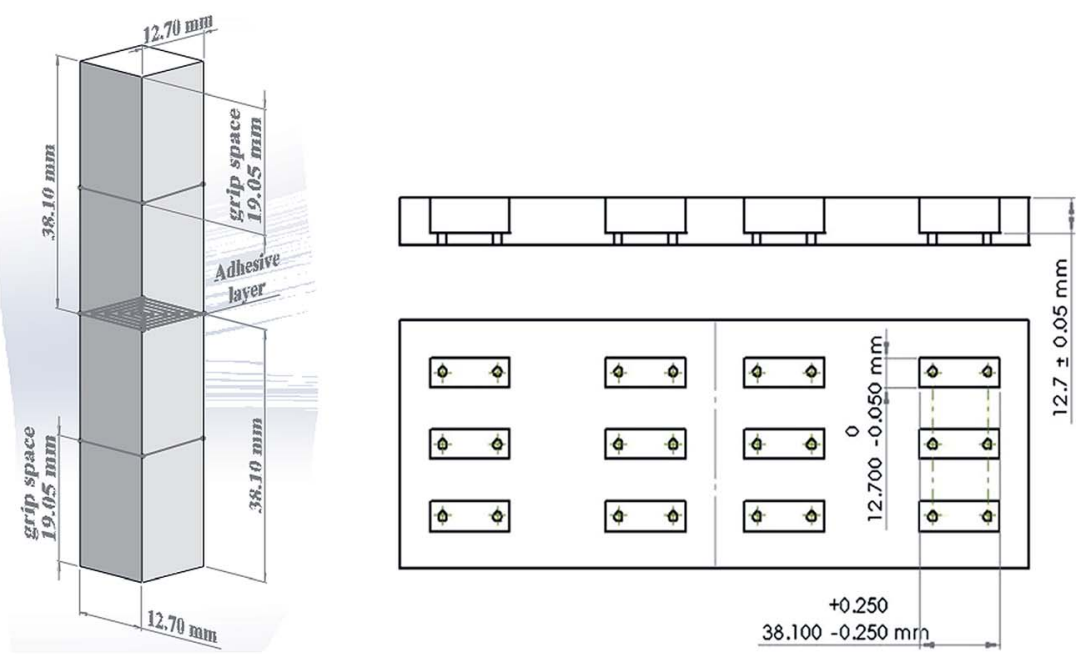

Scheme 1 (a) Schematic of the tensile butt joint strength test specimens (based on ASTM D 2095), (b) schematic of the mold (referred as ASTM D 2095).

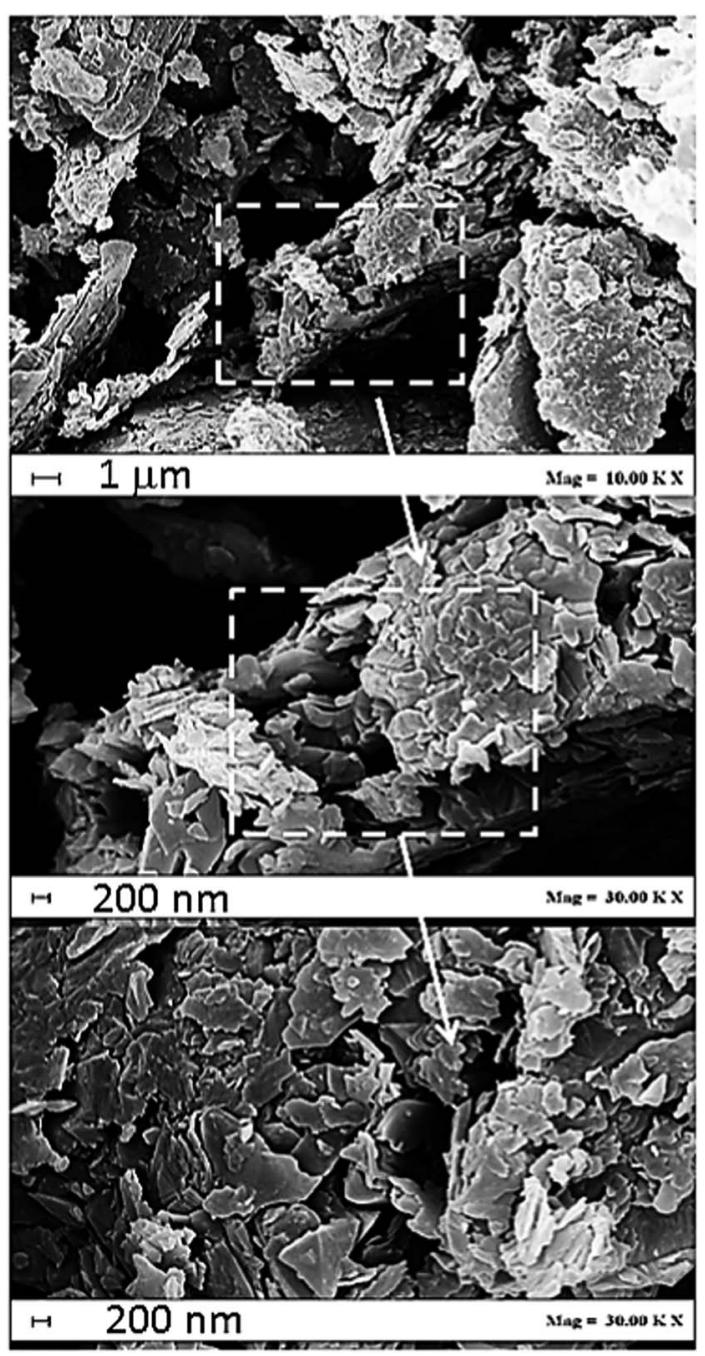

Fig. 1 FESEM images of the platelets of graphene and multilayer graphene platelets with increase in magnification.
To investigate the state of the material that remains dispersed in the supernatant solution after the centrifugation, TEM images have been obtained by dropping a small
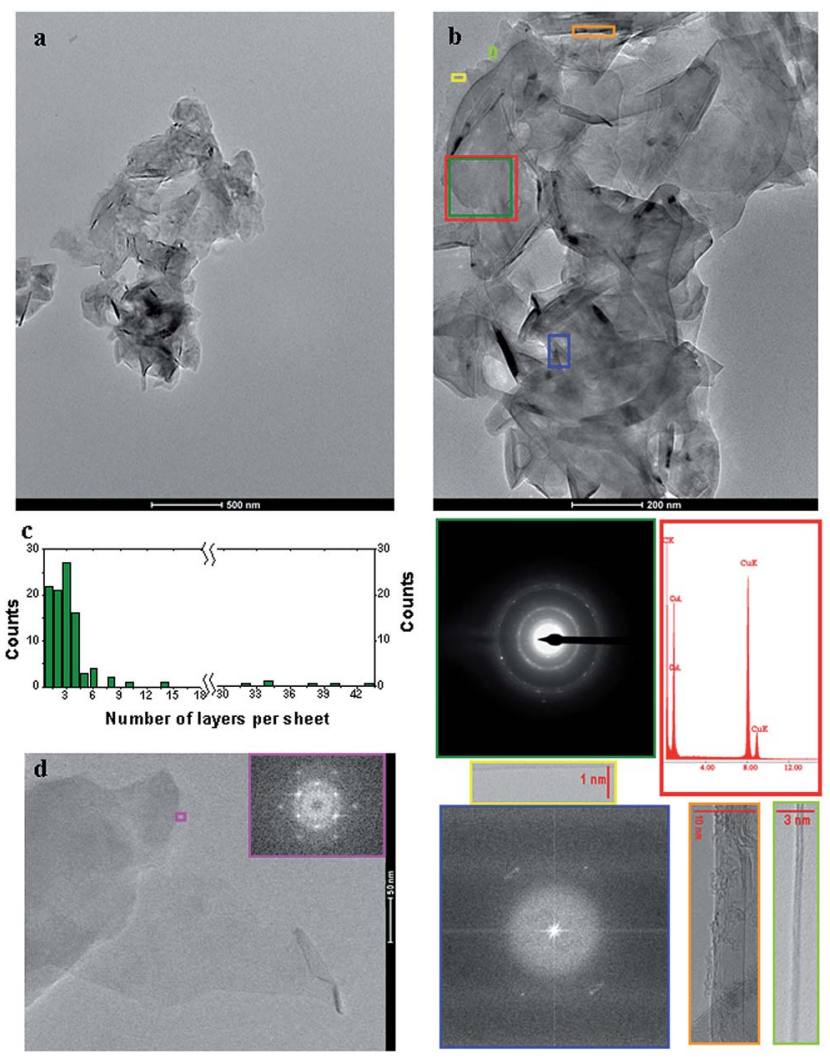

Fig. 2 TEM images at two different magnifications of the platelets of graphene and multilayer graphene platelets ( $a$ and b). The insets in (b) are electron diffraction pattern, in the green area; EDAX analysis, in the red area; FFT, in the blue area; and high resolution TEM images, in the orange, light green and yellow areas. Histogram of the number of layer per platelet (c). High resolution TEM image (d); electron diffraction pattern collected in the pink area of (d). 

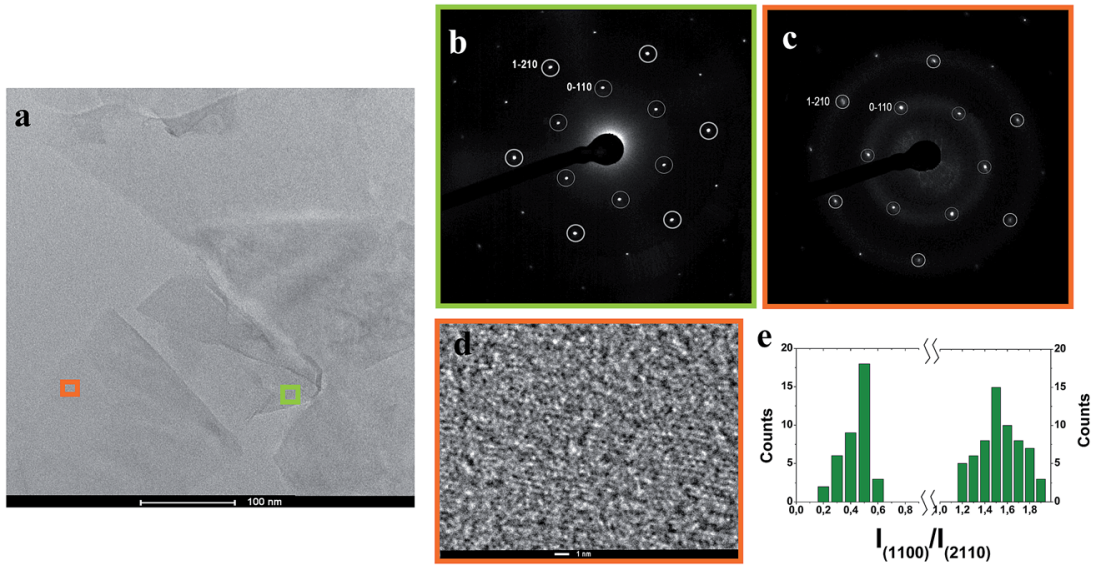

Fig. 3 TEM image of the graphene and multilayer graphene (a); electron diffraction pattern of multilayers in the green area (b); electron diffraction pattern and high resolution TEM image of a monolayer in the orange area (c and d). Histogram of the intensity ratio $I_{(1100)} / I_{(2110)}$ obtained on 100 thin platelets (e)

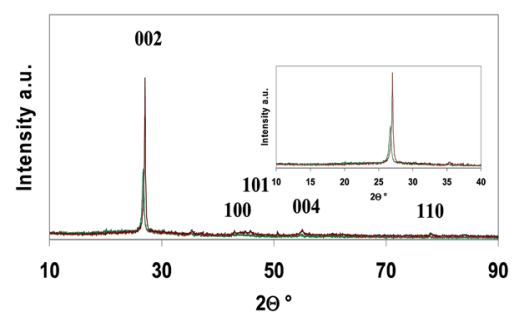

Fig. 4 X-ray diffraction pattern of the platelets of graphene and multilayer graphene platelets, as recorded on the thin film obtained after vacuum filtration.

amount of the dispersion onto holey carbon grids. Fig. 2 shows two typical bright-field TEM images at two different magnifications.

The TEM images (Fig. 2(a) and (b)) of all the samples reveal the presence of platelets of graphene and multilayer graphene platelets with lateral sizes typically of a few micrometers. In many cases, the platelet edges tend to scroll and fold slightly. The corresponding electron diffraction pattern (inset in the green area of Fig. 2(b)) and the EDX spectrum (inset in the red area of Fig. 2(b)) confirm the $\mathrm{sp}^{2}$ nature of the carbon in the platelets. The FFT recorded in the blue area of Fig. 2(b) evidences different orientations of packed multilayer with the typical interlayer graphite spacing of about $0.34 \mathrm{~nm}$. The high resolution TEM images in the insets of Fig. 2(b) allow the counting of the number of layers of the platelets, whose edges are in the areas highlighted by the colored rectangles: 15 and 12 in orange, 2 in light green and 1 in yellow.

By the statistical analysis of over 200 objects from a large number of TEM images, we generated a histogram from the statistics obtained for the thickness of the platelets, as shown in Fig. 2(c) (normalized on 100 objects). From this data, the percentage of monolayer graphene in NMP dispersions can be estimated. We found that the ratio of the number of monolayers/total number of platelets was 0.22 , corresponding to a monolayer mass fraction (mass of monolayers/mass of all the platelets) of 0.055 , leading to an overall yield of $1.65 \mathrm{wt} \%$ (the supernatant contains about $30 \mathrm{wt} \%$ of the original graphite, which was evaluated by TG see Fig. $6 \mathrm{c}$ and the related discussion, further ahead in the text).

In Fig. 2(d), a high resolution TEM image and an electron diffraction pattern, presented in the pink area of the figure, are also reported. The electron diffraction pattern exhibits the typical six-fold symmetry that is expected for graphite/graphene,$^{36}$ confirming the definitive identification of graphene in the pink area.

In particular, a TEM image, showing a number of platelets including a monolayer, is reported in Fig. 3a, together with the electron diffraction patterns obtained in the orange and green area. Labelling the two six-fold symmetry with the MillerBravais $(h k l)$ indices and considering that for the multilayers, the (2110) spots appear to be more intense relative to the (1100) spots (computational studies have shown that the intensity ratio for multilayer, with Bernal (AB) stacking, is $I_{(1100)} / I_{(2110)}<1$, whereas for monolayers it is $I_{(1100)} / I_{(2110)}>1$ (ref. 28)); moreover, we confirm that graphene is present in the orange area and multilayer graphene is present in the green area. Starting from these considerations, we obtain a histogram, reported in Fig. 3e, by measuring the diffraction pattern of 100 thin platelets and the relative intensity ratio $I_{(1100)} / I_{(2110)}$; we find that with respect to the more thinner platelets, the monolayer fraction is about 0.4 .

The XRD diffraction patterns of graphite and G + MLG are reported in Fig. 4.

The characteristic (002) reflection of the samples can be compared to understand the structural changes before and after exfoliation. The peak intensity of pristine graphite is higher than that of $\mathrm{G}+$ MLG, indicating the delamination of graphite into thinner graphene and multilayer graphene platelets. ${ }^{37}$ The lower downshift of the (002) reflection (from $27.0^{\circ}$ to $26.7^{\circ}$ ) indicates a slightly higher interlayer distance (from 3.0 to 3.3 $\mathrm{nm}$ ) between the graphene layers in the platelets after graphite exfoliation. 
a

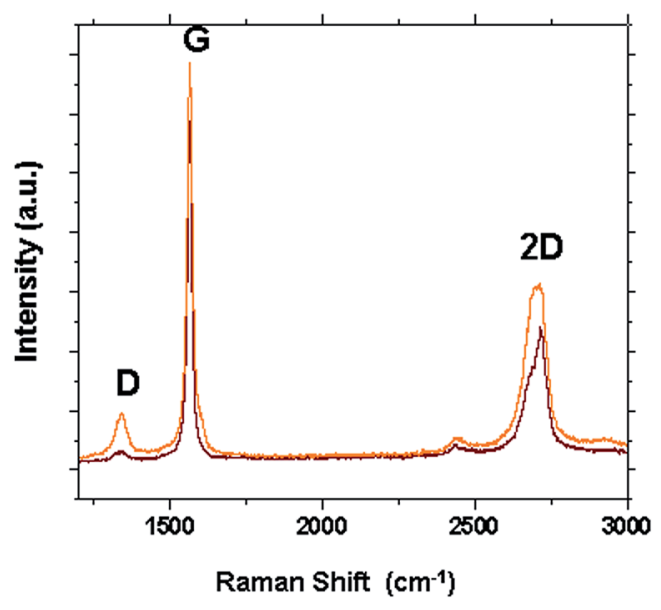

b

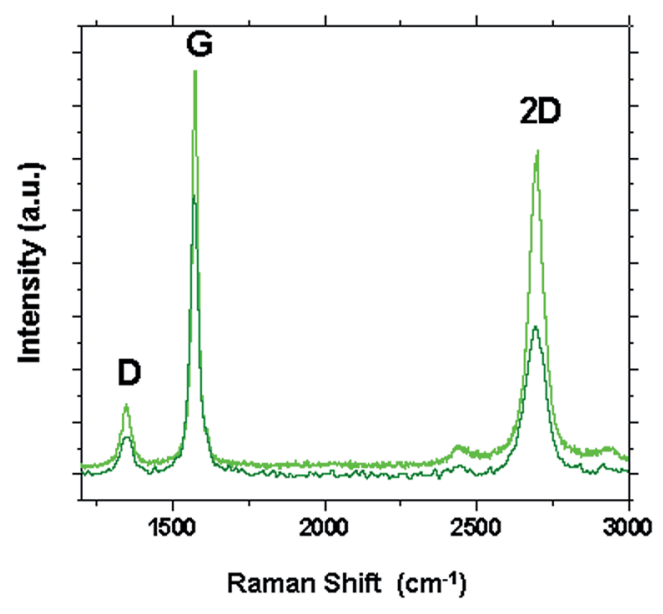

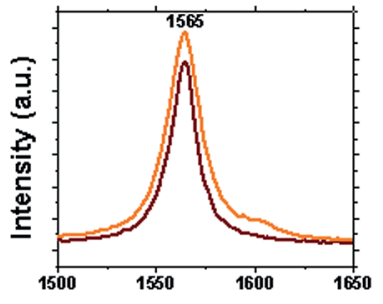

Raman Shift $\left(\mathrm{cm}^{-1}\right)$
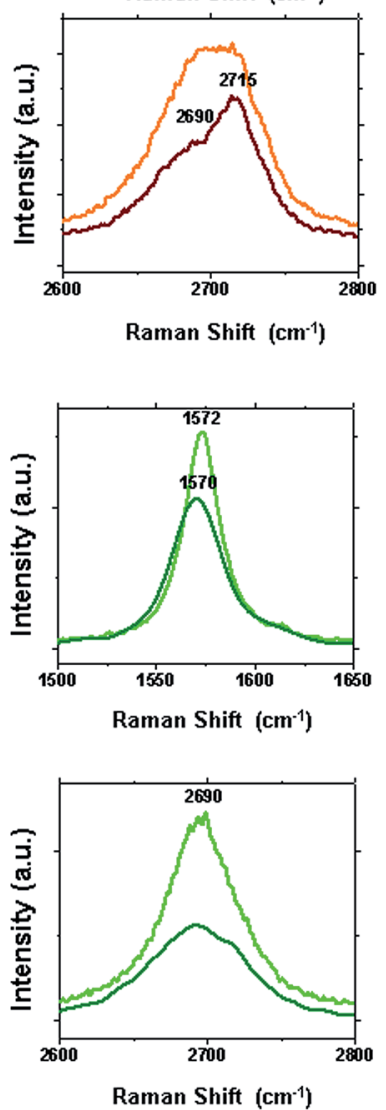

Fig. 5 Raman spectra of the precipitate after centrifugation (a) and the platelets of graphene and multilayer graphene obtained after centrifugation (b).

Fig. 5a shows two typical Raman spectra collected for the precipitate obtained after centrifugation and drying.

The two most intense features are the $G$ peak at $\sim 1570$ $\mathrm{cm}^{-1}$ and a band at $\sim 2700 \mathrm{~cm}^{-1}$, which is named as $2 \mathrm{D}$ because it is the second most prominent peak always observed in graphite samples. ${ }^{38}$ The $G$ peak is doubly degenerate Raman active optical vibration $\mathrm{E}_{2 \mathrm{~g}}$ mode ${ }^{39}$ while the $2 \mathrm{D}$ band is the second order of zone-boundary phonons. These phonons give rise to a peak at about $1350 \mathrm{~cm}^{-1}$ due to a disorder or edge in graphite, which is called as the D band. ${ }^{39}$ The $\mathrm{G}$ band for both the spectra presented in Fig. 5a is centered at $1565 \mathrm{~cm}^{-1}$. The $2 \mathrm{D}$ band in one case (profile in brown) is the typical of graphite, i.e., it consists of two components, namely, 2D1 and 2D2; the second component has a higher intensity than the first. ${ }^{36}$ The profile in orange has the same width as that in brown i.e., a flat apex, and it can be easily deconvoluted with almost two peaks. A broad D- band can be seen in both the spectra which are presented in Fig. 5a. A previous study has demonstrated that this exfoliation process does not introduce a significant structural defect; ${ }^{28}$ however, the laser spot diameter obtained in this investigation was $10 \mu \mathrm{m}$, which is a value higher than the size of the obtained platelets. This suggests the presence of edges within the analyzed area. In addition, the so-called $\mathrm{G}^{*}$ band ${ }^{40}$ can be seen at $2450 \mathrm{~cm}^{-1}$.

Spectra in Fig. 5b were measured for the thin films of $\mathrm{G}+$ MLG, which were prepared by the vacuum filtration of the supernatant onto alumina. Fig. 5b shows a significant change in the shape and intensity of the spectra collected from the supernatant compared to the precipitate. An up-shift of about 7 $\mathrm{cm}^{-1}$ of the $\mathrm{G}$ band and a downshift of the $2 \mathrm{D}$ band, typical of thinner graphite platelets, are observed. ${ }^{41}$

Considering the laser spot size and the platelets dimensions, a certain number of platelets were contemporaneously tested. Thus, 

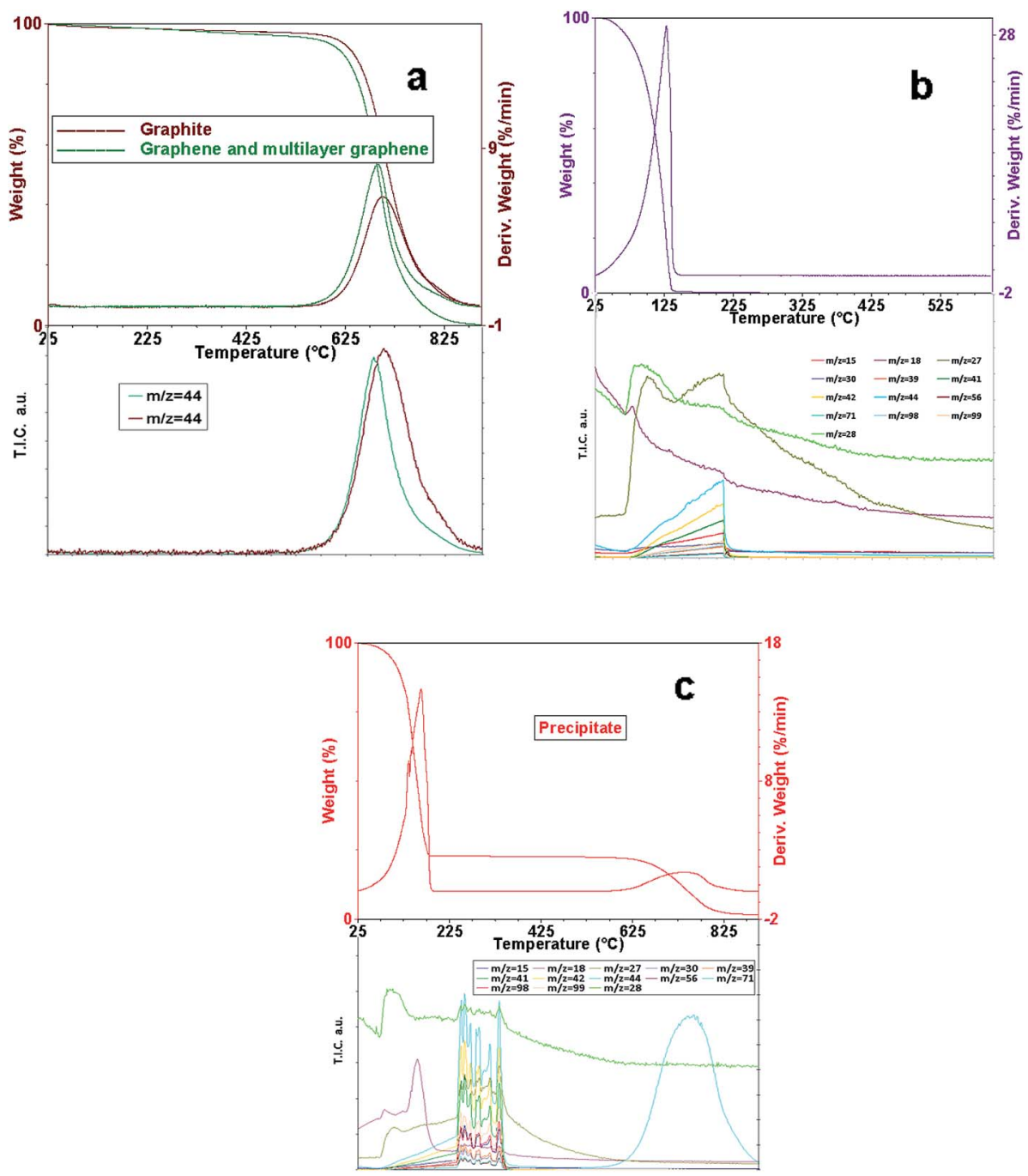

Fig. 6 TG-DTG and relevant total ion current signals of graphite and G + MLG (a). TG-DTG and relevant total ion current signals of NMP (b). TG-DTG and relevant total ion current signals of the precipitate (c).

the Raman spectra collected are the results of the vibration contributions of different platelets. The spectra presented in Fig. 5 are two of the most typical ones. More than 100 spectra have been collected for both the samples, and very few differences were found. What we intend to highlight is the difference between the two phases that are obtained by the graphite exfoliation.

The oxidation process of pristine graphite and G + MLG after drying was compared by thermal analysis (Fig. 6a). The oxidation occurred as a weight loss step in the temperature range of $530-875{ }^{\circ} \mathrm{C}$ with a DTG peak centered at $702{ }^{\circ} \mathrm{C}$ for graphite; these results appeared slightly downshifted to $695{ }^{\circ} \mathrm{C}$ for the obtained sample. The TG residue of the graphite appears likely due to its original purity degree. For both the samples, the oxidation step is characterized by $\mathrm{CO}_{2}$ release (see $m / z=44$ ), while no fragments from NMP or other impurities can be seen during the thermogravimetric test, indicating the high degree of

Table 2 Summary of tensile strength values for all the sets of composite specimens

\begin{tabular}{llll}
\hline Sample label & Adherent & Adhesive & Tensile strength [MPa] \\
\hline A & T20BD & T20BD & $9.090 \pm 1.650$ \\
B & T20BD & T20BD $/ 0.5 \% \mathrm{wt} / \mathrm{wt}$ graphene & $12.74 \pm 4.272$ \\
$\mathrm{C}$ & T20BD & T20BD $/ 1 \% \mathrm{wt} / \mathrm{wt}$ graphene & $18.06 \pm 1.717$ \\
$\mathrm{D}$ & T20BD & T20BD $/ 4 \% \mathrm{wt} / \mathrm{wt}$ graphene & $10.74 \pm 0.217$ \\
$\mathrm{E}$ & T20BD $/ 0.5 \% \mathrm{wt} / \mathrm{wt}$ graphene & T20BD $/ 0.5 \% \mathrm{wt} / \mathrm{wt}$ graphene & $16.98 \pm 0.113$ \\
$\mathrm{~F}$ & T20BD $/ 1 \% \mathrm{wt} / \mathrm{wt}$ graphene & T20BD $/ 1 \% \mathrm{wt} / \mathrm{wt}$ graphene & $18.20 \pm 1.864$ \\
$\mathrm{G}$ & T20BD $/ 4 \% \mathrm{wt} / \mathrm{wt}$ graphene & T20BD $/ 4 \% \mathrm{wt} / \mathrm{wt}$ graphene & $16.47 \pm 0.131$
\end{tabular}




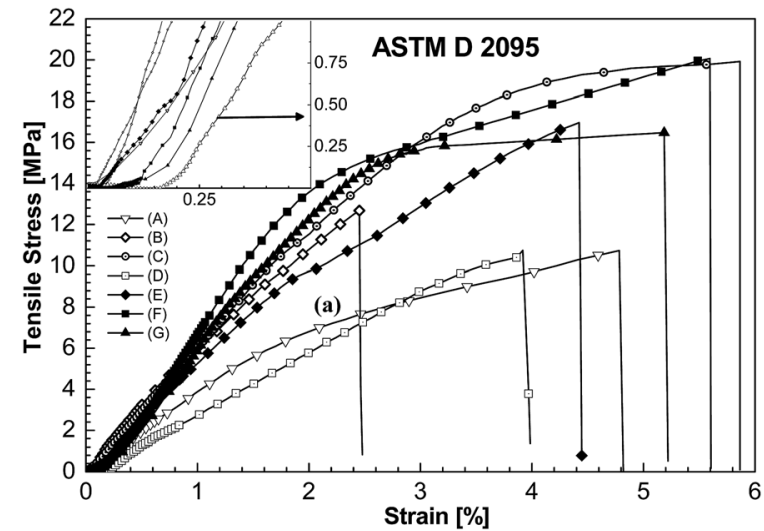

Fig. 7 Stress-strain plot of bonded joints in tensile tests for ASTM D 2095.

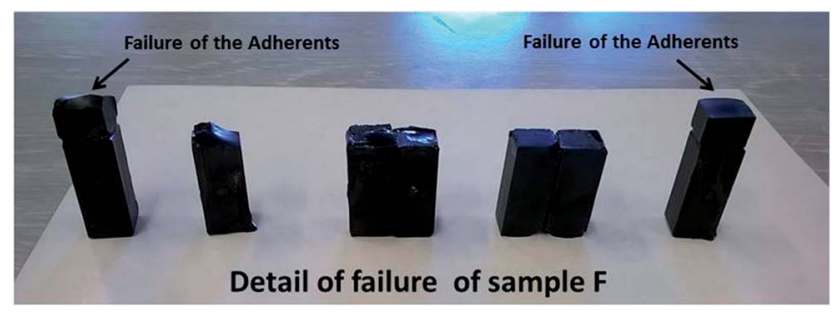

Fig. 8 Detail of failure in sample F.

Table 3 Young's modulus measured on bulk specimens

\begin{tabular}{ll}
\hline Sample label & Young's modulus [MPa] \\
\hline T20BD & $2087.5 \pm 15.57$ \\
T20BD $/ 0.5 \% \mathrm{wt} / \mathrm{wt}$ graphene & $2073.1 \pm 14.90$ \\
T20BD/1\% wt/wt graphene & $2233.8 \pm 54.43$ \\
T20BD/4\% wt/wt graphene & $2274.1 \pm 21.30$
\end{tabular}

purity of the obtained sample; in addition, for the obtained sample, thermogravimetric residue is practically zero.

In Fig. 6b, the thermal conversion of NMP is reported, which shows a weight loss in the range of $25-150{ }^{\circ} \mathrm{C}$; the decomposition of the solvent is clearly indicated by the corresponding TIC of the most intense mass fragment peaks $(\mathrm{m} / \mathrm{z}=$ $15,18,27,28,30,39,41,42,44,56,71,72,98$, and 99). The detector of the spectrometer continues to show the typical fragments of NMP, even after complete oxidation, probably due to the high saturation level of the oxidation chamber.

The thermogravimetric profiles of the precipitate before drying are reported in Fig. 6c. They have been used to evaluate the amount of graphitic carbon in the precipitate, previously opportunely weighed, for a quantitative evaluation. The TG profile shows two weight losses: the first one, in the temperature range of $25-175{ }^{\circ} \mathrm{C}$ is due to NMP, as confirmed by the corresponding TIC of the most intense mass fragment peaks, which are also shown in the Fig. 6; the second one is due to the graphite content. NMP constitutes $75 \mathrm{wt} \%$ of the precipitate. The increased end set temperature is observed probably due to the interaction between the graphite layers and the solvent.

\subsection{Mechanical characterization of adhesive paste}

The average values of the tensile strength of the different bonded joints are listed in Table 2 with their respective standard deviations. In this table, the composition of adherents and adhesive is shown once again to facilitate the text comprehension.

Stress-strain curves are displayed in Fig. 7.

Data in Table 2 highlight that the inclusion of graphene in the adhesive paste up to $1 \% \mathrm{wt} / \mathrm{wt}$ significantly enhances the joint strength (see samples $B$ and $C$ with respect to sample A), leading to a significant improvement in the mechanical behavior of the bonded joint. Nevertheless, for sample $\mathrm{D}$, which corresponds to the highest concentration tested (i.e. $4 \mathrm{wt} / \mathrm{wt} \%$ ), the improvement in the tensile strength value is considerably lower with respect to the samples B and C. It is very likely that this behavior is due to a poor nanofiller dispersion in the adhesive formulation, which is caused by the aggregation of the nanofillers and was confirmed by the morphological analysis of the fracture surface of the bonding areas of sample D (the morphology of the final sample will be discussed later and it has been illustrated in Fig. 11).

The incorporation of graphene as well into the adherents leads to a considerable increase in tensile strength for samples

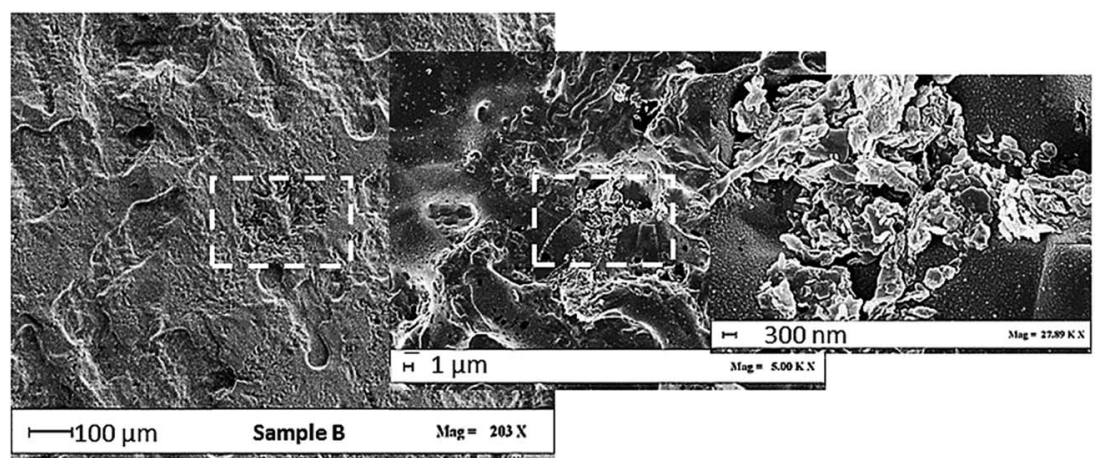

Fig. 9 FESEM micrographs of the fracture surfaces of the bonding areas of sample B. Failure in tensile test and detail of the magnifications of the neighboring region. 


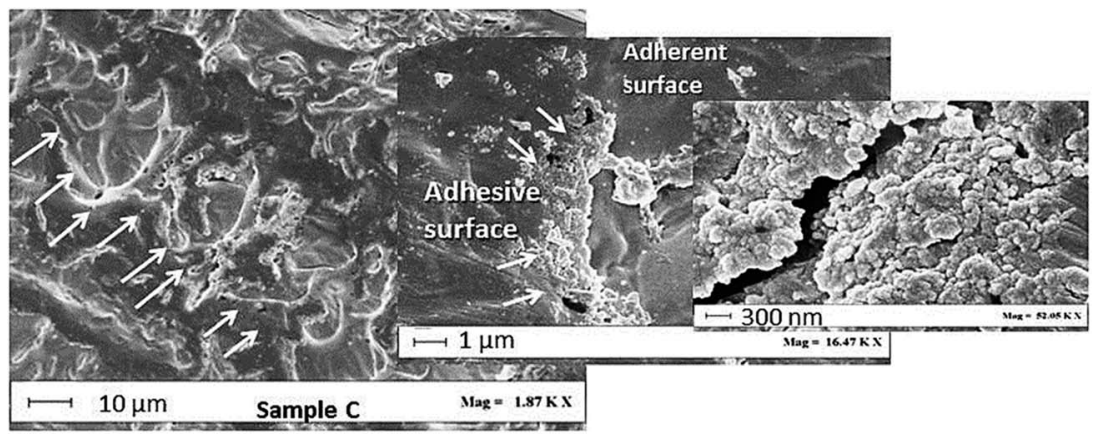

Fig. 10 FESEM micrograph of the fracture surfaces of the bonding areas of sample C. Failure in tensile test and detail of the magnifications of the neighboring region.

E and $\mathrm{G}$ in comparison with the corresponding B and D samples containing unfilled adherents. The comparison of sample C with sample F, which also contains $1 \mathrm{wt} / \mathrm{wt} \%$ of graphene in the adherent, highlights that the sample $\mathrm{F}$ manifests the best performance in the tensile test. In fact, even if only a very slight increase with respect to the corresponding sample $\mathrm{C}$, which contains unfilled adherents, is detected, it is important to highlight that for this sample, replicated tests indicate that the failure mode of the joint occurs in the adherents, as shown in Fig. 8. Therefore, for the sample F, the tensile strength of the bonded area might be potentially higher than the value shown in Table 2. A comprehensive analysis of the results suggests that the incorporation of graphene in the adherents appears to reduce the gap in tensile performance between the samples at different graphene percentages. Furthermore, the higher values detected in the tensile strength of samples $\mathrm{E}, \mathrm{F}$ and $\mathrm{G}$ with respect to $\mathrm{B}, \mathrm{C}$ and $\mathrm{D}$ highlight that in the case of joints containing graphene platelets embedded in the adherents, the failure between the adhesive and adherents is more hindered than the joints with unfilled adherents. This is a clear indication that graphene platelets at the interfaces between the adhesive paste and adherents act in such a manner that they strengthen the attractive intermolecular forces.

Young's modulus measured on bulk specimens are reported in Table 3. It slightly increases with increasing graphene concentration.
Young's modulus increases from $2087 \mathrm{MPa}$ for the unfilled resin to $2274 \mathrm{MPa}$ for the filled resin (4\% wt/wt graphene), exhibiting an increase of approximately $9 \%$.

\subsection{Morphological analysis}

Fig. 9-14 show the FE-SEM micrographs of the fracture surface of the samples tested in the tensile mode. For each specimen, three types of micrographs are presented: one at a relatively low magnification and others at higher magnifications. The morphological investigation of the fracture surface of butt joints highlights that the difference in the mechanical performance of the bonded joints in tensile tests is closely correlated to the different distribution of the graphene in the epoxy matrix. The etched fracture surfaces of the bonding areas of samples B and C (see Fig. 9 and 10) show a failure in the adhesive part in the majority of the surface areas of the bonded parts. For both the samples, in fact, the mechanism of the failure of bonded joint occurs into the layer of adhesive that remains on both the adherent surfaces. The higher value of mechanical strength achieved for these sample with respect to sample A is effectively due to the transfer of the external load to the adhesive, which contains well dispersed graphene nanoplatelets that behave as the strongest part of the composite adhesive. Nevertheless, at a higher nanofiller content (i.e. $4 \% \mathrm{wt} / \mathrm{wt}$ ) (see sample D, Fig. 11), nanofiller aggregates of several microns cause a strong heterogeneity in the morphological feature of the resin. This

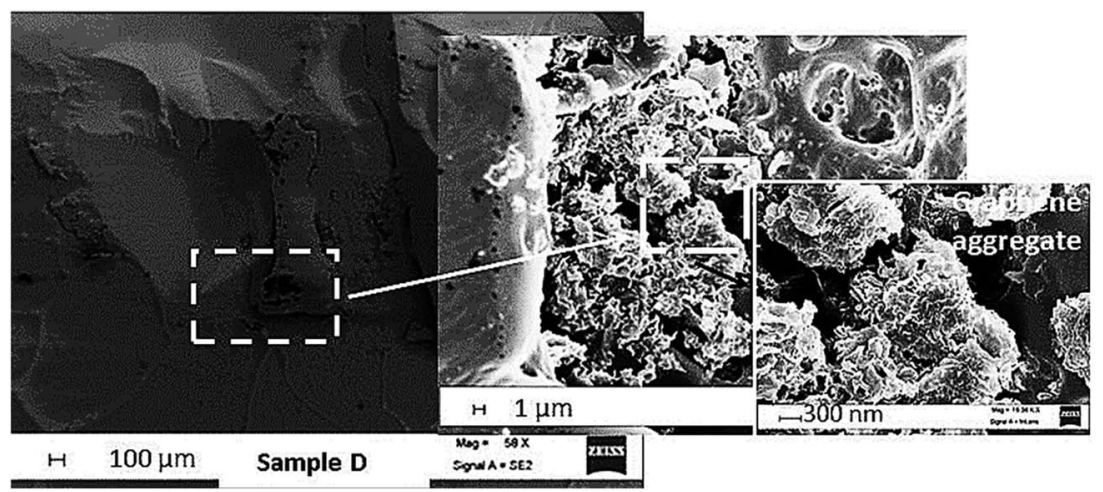

Fig. 11 FESEM micrograph of the fracture surfaces of the bonding areas of sample D. Failure in tensile test and detail of the magnifications of the neighboring region. 


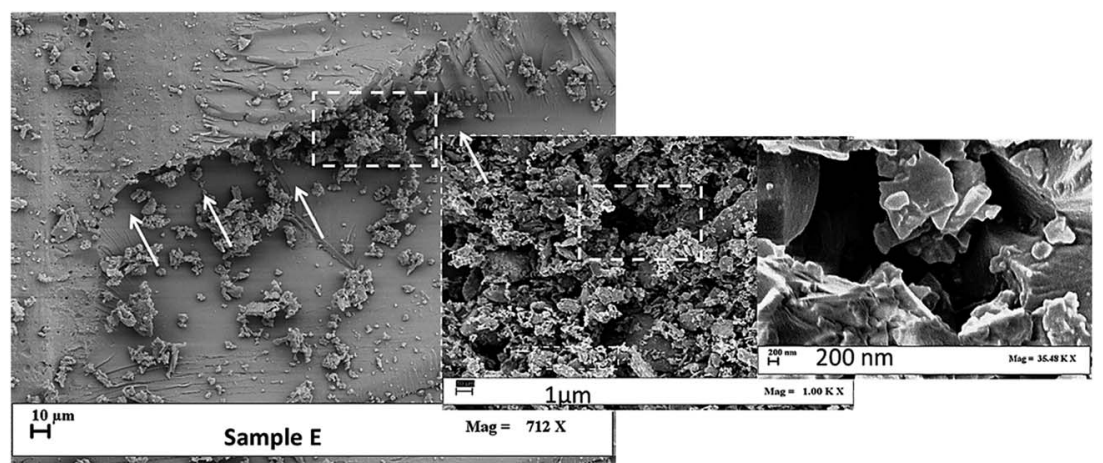

Fig. 12 FESEM micrograph of the fracture surfaces of the bonding areas of sample E. Failure in tensile test and detail of the magnifications of the neighboring region.

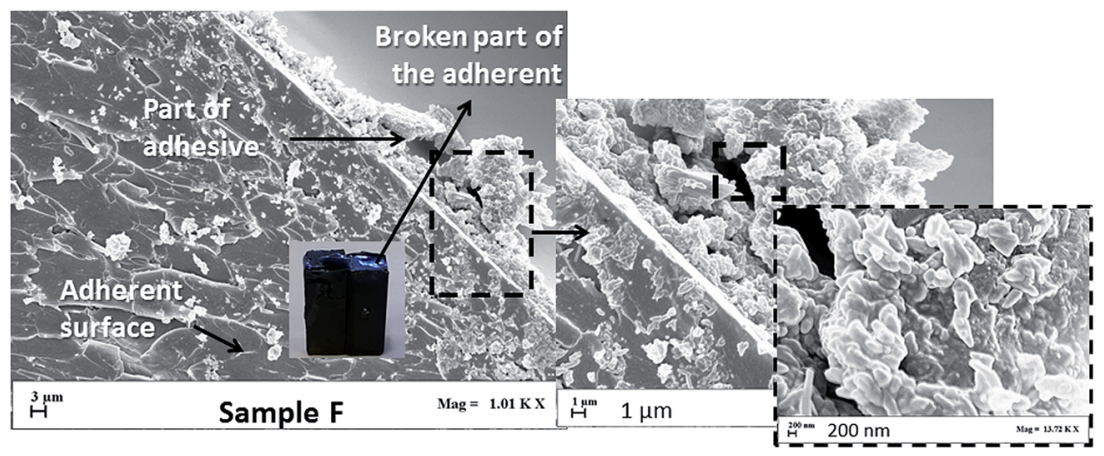

Fig. 13 FESEM micrograph of the fracture surfaces of the bonding areas of sample F. Failure in tensile test and detail of the magnifications of the neighboring region.

occurrence is most likely to be responsible for the reduced value for the improvement in the tensile strength of joint with respect to the expected value.

The distribution of the graphene platelets in the epoxy matrix plays a crucial role in the adhesion. In fact, the tensile strength increases with the filler concentration, until at a higher concentration, the formation of aggregates causes discontinuity in the stress transfer inside the sample with a consequent decrease in the mechanical performance of the joint.

In the case of joints composed of filled adherents and filled epoxy paste (Fig. 12-14), it is possible to observe a failure in the adhesive part for sample E (see Fig. 12), which shows a good dispersion of graphene platelets in the adhesive paste that

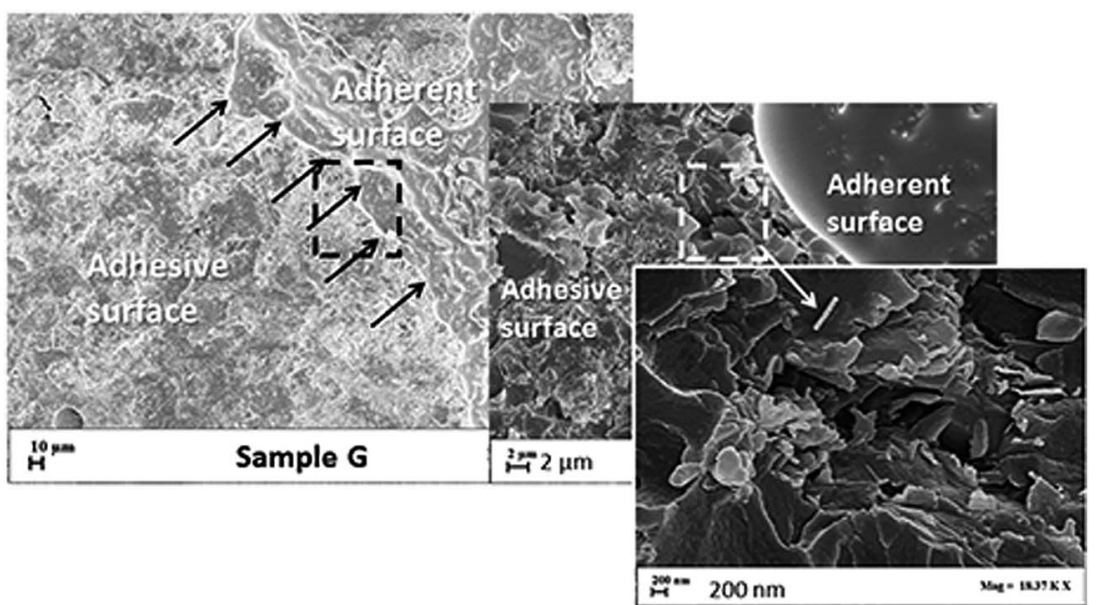

Fig. 14 FESEM micrograph of fracture surfaces of the bonding areas of sample G. Failure in tensile test and detail of the magnifications of the neighboring region. 
covers both the surfaces of the adherents. For sample F, presented in Fig. 13, it is possible to observe a good dispersion of graphene platelets in the adherents and adhesive paste due to the fact that the failure involves a large part of adherent material, as already described in the previous section; the failure in the adherents could be a signal of good adhesion.

For sample G (see Fig. 14), it is possible to observe clean parts of adherents, which are indicative of adhesive failure in some regions (see the part of adherents surfaces) at the interface between adhesive and adherents (see the region indicated as "part of adherents surfaces"). The lower value of tensile strength with respect to the other joints of the same series could be due to the regions in which the nanofiller aggregates facilitate an easier debonding between some zones of adhesive and adherents.

The improvement in mechanical properties achieved with graphene platelets in the adhesive and adherents could be due to the intermolecular interactions such as intermolecular hydrogen bonding between the hydroxyl or carboxylic groups of graphene platelets and cross-linked epoxy resins. This hypothesis appears to be confirmed by the additional benefit that is provided to the tensile strength by the joints filled with graphene platelets.

\section{Conclusions}

Graphene and multilayer graphene have been prepared by a liquid phase exfoliation in $N$-methylpyrrolidone (NMP). The graphite concentration and the sonication conditions (ultrasound time and power) allowed to obtain in a one step a massive amount of very pure thin platelets (exfoliated graphite $30 \mathrm{wt} \%$ of the original graphite, TG evaluation) with a monolayer yield of about $1.8 \mathrm{wt} \%$.

The reinforcement effect due to graphene platelets has proven to be very effective in improving the attractive interaction between the adhesive and adherent surface. In fact, the addition of an appropriate amount of graphene platelets into an epoxy adhesive formulation designed for structural application caused a significant improvement in the mechanical performance of the joints. An amount equal to or higher than $4 \% \mathrm{wt}$ / wt was observed to be deleterious for the adhesive properties. The adverse effect is more probably due to the aggregations and then poor dispersion of the nanofiller into the adhesive paste. A further improvement in the mechanical performance of the joints is achieved by adding graphene platelets as well in the adherents. This beneficial effect could be due to the cumulative joined effects of intermolecular interactions such as intermolecular hydrogen bonding between the hydroxyl or carboxylic groups of graphene platelets and $\mathrm{OH}$ groups of cross-linked epoxy resins. The application of this strategy to increase the attractive forces at the interphase between the adhesive and adherents has the potential to open new scopes and opportunities in the design of innovative joints, allowing to expand the use of the adhesive bonding of structural parts, thus increasing the competitiveness of the industries in terms of performance and cost. It is worth noting that the inclusion of conductive nanofiller in adhesives for composite structures has also the prospective to obtain multifunctional adhesives, which are able to simultaneously integrate the structural mechanical performance and the required electrical conductivity. The appropriate selection and modification of the polymer matrix composition, the nanofiller type and the control of the interactions at the interface can allow for obtaining tailored multifunctional properties of the resulting adhesive, which could otherwise not be achieved by conventional materials or composites.

\section{Acknowledgements}

This research has received funding from the European Union's Seventh Framework Programme for research, technological development and demonstration under grant agreement no. 313978.

\section{References}

1 L. Guadagno, M. Raimondo, K. Lafdi, A. Fierro, S. Rosolia and M. R. Nobile, Influence of nanofiller morphology on the viscoelastic properties of CNF/epoxy resins, AIP Conf. Proc., 2014, 1599, 386-389.

2 L. Guadagno, M. Raimondo, U. Vietri, L. Vertuccio, G. Barra, B. De Vivo, P. Lamberti, G. Spinelli, V. Tucci, R. Volponi, G. Cosentino and F. De Nicola, Effective Formulation and Processing of Nanofilled Carbon Fiber Reinforced Composites, RSC Adv., 2015, 5, 6033-6042.

3 L. Guadagno, M. Raimondo, V. Vittoria, L. Vertuccio, C. Naddeo, P. Lamberti and V. Tucci, Resina epossidica con basso tenore di umidità, Italian Patent no. TO2013A000926, 15 November 2013.

4 L. Guadagno, M. Raimondo, V. Vittoria, L. Vertuccio, C. Naddeo, S. Russo, B. De Vivo, P. Lamberti, G. Spinelli and V. Tucci, Development of epoxy mixtures for application in aeronautics and aerospace, RSC Adv., 2014, 4, 15474-15488.

5 K. T. Hsiao, J. Alms and S. G. Advani, Use of epoxy/ multiwalled carbon nanotubes as adhesives to join graphite fiber reinforced polymer composites, Nanotechnology, 2003, 14, 791-793.

$6 \mathrm{~S}$. Yu, M. N. Tong and G. Critchlow, Wedge test of carbonnanotube-reinforced epoxy adhesive joints, J. Appl. Polym. Sci., 2009, 111, 2957-2962.

7 S. A. Meguid and Y. Sun, On the tensile and shear strength of nano-reinforced composite interfaces, Mater. Des., 2004, 25, 289-296.

8 U. Vietri, L. Guadagno, M. Raimondo, L. Vertuccio and K. Lafdi, Nanofilled epoxy adhesive for structural aeronautic materials, Composites, Part B, 2014, 61, 73-83.

9 A. S. Neto, D. T. L. Da Cruz and A. F. Ávila, Nano-modified adhesive by graphene: The single lap-joint case, Mater. Res., 2013, 16, 592-596.

10 F. H. Gojny, M. H. G. Wichmann, B. Fiedler, W. Bauhofer and K. Schulte, Influence of nano-modification on the mechanical and electrical properties of conventional fibrereinforced composites, Composites, Part A, 2005, 36, 15251535. 
11 T. Wang, C. H. Lei, A. B. Dalton, C. Creton, Y. Lin, K. A. S. Fernando, Y. P. Sun, M. Manea, J. M. Asua and J. L. Keddie Waterbone, Nanocomposite Pressure-Sensitive Adhesives with High Tack Energy, Optical Transparency, and Electrical Conductivity, Adv. Mater., 2006, 18, 27302734 .

12 C. W. Nan, Y. Shen and J. Ma, Physical Properties of Composites Near Percolation, Annu. Rev. Mater. Res., 2010, 40, 131-151.

13 L. Guadagno, B. De Vivo, A. Di Bartolomeo, P. Lamberti, A. Sorrentino, V. Tucci, L. Vertuccio and V. Vittoria, Effect of functionalization on the thermo-mechanical and electrical behavior of multi-wall carbon nano tube/epoxy composites, Carbon, 2011, 49, 1919-1930.

14 B. De Vivo, L. Guadagno, P. Lamberti, M. Raimondo, G. Spinelli, V. Tucci, L. Vertuccio and V. Vittoria, Electrical properties of multi-walled carbon nano tube/ tetrafunctional epoxy-amine composites, AIP Conf. Proc., 2012, 1459, 199-201.

15 L. Guadagno, M. Raimondo, V. Vittoria, L. Vertuccio, K. Lafdi, B. De Vivo, P. Lamberti, G. Spinelli and V. Tucci, The role of carbon nanofiber defects on the electrical and mechanical properties of CNF-based resins, Nanotechnology, 2013, 24, 305704.

16 S. G. Prolongo, M. R. Gude and A. Ureña, Nanoreinforced Adhesives, in Nanotechnology and Nanomaterials "Nanofibers", ed. Ashok Kumar, InTech, 2010, ch. 3, pp. 39-68, ISBN 978-953-7619-86-2.

17 A. K. Geim and K. S. Novoselov, The rise of graphene, Nat. Mater., 2007, 6, 183-191.

18 T. J. Echtermeyer, M. C. Lemme, M. Baus, B. N. Szafranek, A. K. Geim and H. Kurz, Nonvolatile switching in graphene field-effect devices, IEEE Electron Device Lett., 2008, 29, 952-954.

19 G. Eda, G. Fanchini and M. Chhowalla, Large-area ultrathin films of reduced graphene oxide as a transparent and flexible electronic material, Nat. Nanotechnol., 2008, 3, 270-274.

20 K. S. Kim, Y. Zhao, H. Jang, S. Y. Lee, J. M. Kim, K. S. Kim, J. H. Ahn, P. Kim, J. Y. Choi and B. H. Hong, Large-scale pattern growth of graphene films for stretchable transparent electrodes, Nature, 2009, 457, 706-710.

21 P. Blake, P. D. Brimicombe, R. R. Nair, T. J. Booth, D. Jiang, F. Schedin, L. A. Ponomarenko, S. V. Morozov, H. F. Gleeson, E. W. Hill, A. K. Geim and K. S. Novoselov, Graphene-based liquid crystal device, Nano Lett., 2008, 8, 1704-1708.

22 M. D. Stoller, S. Park, Y. Zhu, J. An and R. S. Ruoff, GrapheneBased Ultracapacitors, Nano Lett., 2008, 8, 3498-3502.

23 D. A. Dikin, S. Stankovich, E. J. Zimney, R. D. Piner, G. H. B. Dommett, G. Evmenenko, S. T. Nguyen and R. S. Ruoff, Preparation and characterization of graphene oxide paper, Nature, 2007, 448, 457-460.

24 J. S. Bunch, S. S. Verbridge, J. S. Alden, A. M. van der Zande, J. M. Parpia, H. G. Craighead and P. L. McEuen, Nano Lett., 2008, 8, 2458-2462.

25 N. Yan, G. Buonocore, M. Lavorgna, S. Kaciulis, S. K. Balijepalli, Y. Zhan, H. Xia and L. Ambrosio, The role of reduced graphene oxide on chemical, mechanical and barrier properties of natural rubber composites, Compos. Sci. Technol., 2014, 102, 74-81.

26 G. Scherillo, M. Lavorgna, G. G. Buonocore, Y. H. Zhan, H. S. Xia, G. Mensitieri and L. Ambrosio, Tailoring assembly of reduced graphene oxide nanosheets to control gas barrier properties of natural rubber nanocomposites, ACS Appl. Mater. Interfaces, 2014, 6, 2230-2234.

27 S. Stankovich, D. A. Dikin, R. D. Piner, K. A. Kohlhaas, A. Kleinhammes, Y. Jia, Y. Wu, S. T. Nguyen and R. S. Ruoff, Synthesis of graphene-based nanosheets via chemical reduction of exfoliated graphite oxide, Carbon, 2007, 45, 1558-1565.

28 Y. Hernandez, V. Nicolosi, M. Lotya, F. M. Blighe, Z. Sun, S. De, I. T. McGovern, B. Holland, M. Byrne, Y. K. Gun'ko, J. J. Boland, P. Niraj, G. Duesberg, S. Krishnamurthy, R. Goodhue, J. Hutchison, V. Scardaci, A. C. Ferrari and J. N. Coleman, High-yield production of graphene by liquid-phase exfoliation of graphite, Nat. Nanotechnol., 2008, 3, 563-568.

29 A. Reina, X. Jia, J. Ho, D. Nezich, H. Son, V. Bulovic, M. S. Dresselhaus and J. Kong, Large Area, Few-Layer Graphene Films on Arbitrary Substrates by Chemical Vapor Deposition, Nano Lett., 2009, 9, 30-35.

30 Y. Lee, S. Bae, H. Jang, S. Jang, S. E. Zhu, S. H. Sim, Y. I. Song, B. H. Hong and J. H. Ahn, Wafer-Scale Synthesis and Transfer of Graphene Films, Nano Lett., 2010, 10, 490-493.

31 M. Sarno, C. Cirillo, R. Piscitelli and P. Ciambelli, A study of the key parameters, including the crucial role of $\mathrm{H}_{2}$ for uniform graphene growth on Ni foil, J. Mol. Catal. A: Chem., 2013, 366, 303-314.

32 M. Sarno, C. Cirillo and P. Ciambelli, Selective graphene covering of monodispersed magnetic nanoparticles, Chem. Eng. J., 2014, 246, 27-38.

33 M. Funaro, M. Sarno, P. Ciambelli, C. Altavilla and A. Proto, Real time radiation dosimeters based on vertically aligned multiwall carbon nanotubes and graphene, Nanotechnology, 2013, 24, 075704.

34 Z. Chen, W. Ren, B. Liu, L. Gao, S. Pei, Z. S. Wu, J. Zhao and H. M. Cheng, Bulk-growth of mono-to few-layer graphene on nickel particles by chemical vapor deposition from methane, Carbon, 2010, 48, 3543-3550.

35 M. Sarno, A. Senatore, C. Cirillo, V. Petrone and P. Ciambelli, Oil lubrificant tribological behaviour improvement through dispersion of few layer graphene oxide, J. Nanosci. Nanotechnol., 2014, 14, 4960-4968.

36 J. C. Meyer, A. K. Geim, M. I. Katsnelson, K. S. Novoselov, T. J. Booth and S. Roth, The structure of suspended graphene sheets, Nature, 2007, 446, 60-63.

37 S. Y. Bae, I. Y. Jeon, J. Yang, N. Park, H. Suk Shin, S. Park, R. S. Ruoff, L. Dai and J. B. Baek, Large-Area Graphene Films by Simple Solution Casting of Edge-Selectively Functionalized Graphite, ACS Nano, 2011, 5, 4974-4980.

38 C. Casiraghi, A. C. Ferrari and J. Robertson, Raman Spectroscopy of hydrogenated amorphous carbons, Phys. Rev. B: Condens. Matter Mater. Phys., 2005, 72, 085401. 
39 A. C. Ferrari, S. E. Rodil and J. Robertson, Interpretation of infrared and Raman spectra of amorphous carbon nitrides, Phys. Rev. B: Condens. Matter Mater. Phys., 2003, 67, 155306.

40 L. M. Malard, M. A. Pimenta, G. Dresselhaus and M. S. Dresselhaus, Raman Spectroscopy in graphene, Phys. Rep., 2009, 473, 51-87.
41 A. C. Ferrari, Raman spectroscopy of graphene and graphite: disorder, electron-phonon coupling, doping and nonadiabatic effects, Solid State Commun., 2007, 143, 47-57. 